\title{
NGC 2180: A disrupting open cluster?
}

\begin{abstract}
C. Bonatto, E. Bica, and D. B. Pavani
Universidade Federal do Rio Grande do Sul, Instituto de Física, CP 15051, Porto Alegre 91501-970, RS, Brazil

e-mail: charles@if.ufrgs.br

Received 16 March 2004 / Accepted 18 July 2004

Abstract. The spatial dependence of luminosity and mass functions of evolved open clusters is discussed in this work using $J$ and $H$ 2MASS photometry, which allows a wide spatial coverage and proper background determination. The target objects are the intermediate-age open cluster NGC $3680\left(\ell=286.76^{\circ}\right.$ and $\left.b=16.92^{\circ}\right)$, which has been reported as being in an advanced state of dissolution, and $\operatorname{NGC} 2180\left(\ell=203.85^{\circ}, b=-7.01^{\circ}\right)$, a possible dynamically evolved open cluster. At the $1-\sigma$ level, NGC 2180 presents a radial distribution of stars that can be represented by a King law. We conclude that, although in an advanced dynamical state (mass segregated), NGC 3680 does not present strong signs of dissolution, having luminosity and mass functions very similar to those of the $\tau_{\text {age }} \approx 3.2$ Gyr open cluster M 67. On the other hand, NGC 2180 appears to have flat, eroded luminosity functions throughout its structure, suggesting that in addition to mass segregation, Galactic tidal stripping has been effective in depleting this object. Accordingly, NGC 2180 may be the missing link between evolved open clusters and remnants. For NGC 2180 we infer a distance to the Sun $d_{\odot}=0.91 \pm 0.15 \mathrm{kpc}$, an age $\tau_{\text {age }} \approx 710 \mathrm{Myr}$ and an observed stellar mass of $m_{\mathrm{obs}} \sim 47 M_{\odot}$. For NGC 3680 we derive an age $\tau_{\mathrm{age}} \approx 1.6 \mathrm{Gyr}, E(B-V)=0.0$ and $d_{\odot}=1.00 \pm 0.09 \mathrm{kpc}$, confirming previous estimates. The observed stellar mass $m_{\mathrm{obs}} \approx 130 M_{\odot}$ agrees with previous values. We study both clusters in the context of dynamical states estimated from diagnostic diagrams involving photometric and structural parameters. Both objects appear to be dynamically evolved systems. In particular, NGC 2180 is closer to open cluster remnants than NGC 3680.
\end{abstract}

Key words. Galaxy: open clusters and associations: general - stars: luminosity function, mass function

\section{Introduction}

Open clusters are formed along the gas and dust-rich Galactic plane and contain from tens to a few thousands stars distributed in an approximately spherical structure of up to a few parsecs in radius. This loose condition makes them potentially short-lived stellar systems, since disruptions may occur by the cumulative effect of passages near interstellar clouds and/or by shocks with the Galactic disk. Cumulative orbital perturbations may lead to more internal orbits, enhancing such effects (Bergond et al. 2001). Consequently, most of the open clusters in the Galaxy evaporate completely in less than $1 \mathrm{Gyr}$. Indeed, the open cluster catalogue of Lyngå (1987) indicated about 70 objects older than $1 \mathrm{Gyr}(\approx 6 \%$ of the total number).

The dynamical evolution of an open cluster depends both on internal and external factors. Internal factors are: $(i)$ after successive 2-body encounters with more massive stars, lessmassive stars may acquire velocities larger than the cluster's escape velocity; and (ii) the normal stellar evolution via massloss. The external factors are: (i) large-scale encounters with giant molecular clouds (Wielen 1991); and (ii) tidal stripping by the Galactic gravitational field. A typical open cluster at the solar radius will cross the Galactic plane 10-20 times before being disrupted and leaving an open cluster remnant (de la Fuente Marcos 1998). Bergond et al. (2001) estimate the destruction time-scale for open clusters in the solar neighbourhood at about $600 \mathrm{Myr}$. Consequently, it is expected that only those open clusters which are born with the largest masses or those located at large Galactic radii will survive longer than a few Gyr (Friel 1995).

The numerical simulations of de la Fuente Marcos (1996) have shown that the final cluster remnant composition depends on the initial mass function, fraction of primordial binaries and galactocentric distance. The resulting cluster remnants are rich in binaries and do not appear to contain collapsed objects. Remnants of poorly populated clusters are expected to contain early-type stars, while those of more massive clusters contain late-type stars (de la Fuente Marcos 1996), owing to different evolutionary time-scales. In the central region of the more evolved clusters, mass segregation should deplete the low main-sequence (MS) stars thus creating a core rich in compact and giant stars (Takahashi \& Portegies Zwart 2000).

Mass segregation in a star cluster scales with the relaxation time, defined as $t_{\text {relax }}=\frac{N}{8 \ln N} t_{\text {cross }}$, where $t_{\text {cross }}=R / v$ is the crossing time (Binney \& Tremaine 1987). For a typical cluster radius of $R \sim 5 \mathrm{pc}$ and velocity dispersion $v \sim 1 \mathrm{~km} \mathrm{~s}^{-1}, t_{\text {relax }} \sim$ $13 \mathrm{Myr}$ for a cluster with $N=10^{2}$ stars, and $t_{\text {relax }} \sim 90 \mathrm{Myr}$ for $N=10^{3}$ stars.

Recently, several studies called attention to the possibility of detecting open cluster remnants in the Galaxy, e.g. Bica et al. (2001), Carraro (2002), Pavani et al. (2002, 2003). A fundamental question to dynamical evolution studies is 
whether any open cluster can be observed right at the disrupting phase, when the remaining low-mass stars in the cluster's halo get dispersed into the background and the corresponding mass function becomes eroded.

Depletion of low-MS stars in the central parts of a cluster is a sign of advanced dynamical evolution. This has been detected e.g. in NGC 3680 (Anthony-Twarog et al. 1991) and M 67 (Bonatto \& Bica 2003), in which the presence of a corona rich in low-mass stars has been confirmed with 2MASS photometry.

The Two Micron All Sky Survey (hereafter 2MASS, Skrutskie et al. 1997) has proven to be a powerful tool in the analyses of the structure and stellar content of open clusters (e.g. Bonatto \& Bica 2003; Bica et al. 2003). Indeed, the uniform and essentially complete sky coverage provided by 2MASS allows one to properly take into account background regions with suitable star count statistics, which is fundamental in order to correctly identify and characterize the stellar content of clusters, since their ages and distances can be determined by fitting isochrones to their colour-magnitude diagrams (CMDs), with a precision depending on the depth of the photometry and field contamination.

In the present study we address the actual dynamical state of NGC 3680 analysing a large spatial area in the direction of the cluster, which 2 MASS can provide. In particular, we search for the presence of a low-mass star-rich corona. In addition, we discuss NGC 2180, an overlooked open cluster. This cluster appears to be in a more advanced dynamical evolutionary stage than NGC 3680, and thus might be a missing link between evolved open clusters with a corona and final remnants.

In Sect. 2 we provide available information on NGC 2180 and the intermediate-age open cluster NGC 3680. In Sect. 3 we obtain the 2MASS photometry and introduce the $J \times(J-H)$ CMDs. In Sect. 4 we discuss the radial density distribution of stars and derive structural parameters for the clusters. In Sect. 5 we fit isochrones to the near-infrared CMDs and derive cluster parameters. In Sect. 6 we derive the luminosity and mass functions (hereafter LF and MF) and estimate the stellar masses of each cluster. In Sect. 7 we compare NGC 2180 and NGC 3680 with well-known dynamically evolved open clusters and open cluster remnants. In Sect. 8 we present and discuss proper motions in the direction of both objects. Concluding remarks are given in Sect. 9.

\section{The target objects}

\subsection{NGC2180: A W. Herschell's overlooked open cluster?}

NGC 2180 was first observed and described as a cluster by W. Herschell (Dreyer 1888). It is not included in modern catalogues of open clusters (Alter et al. 1970; Lyngå 1987). In Dias et al. (2002) and in a revision of the NGC catalogue (Sulentic \& Tifft 1973), NGC 2180 is indicated as non-existing. Houston (1976) included it in a list of possible clusters. The only information currently available in the WEBDA open cluster database (Mermilliod 1996 -

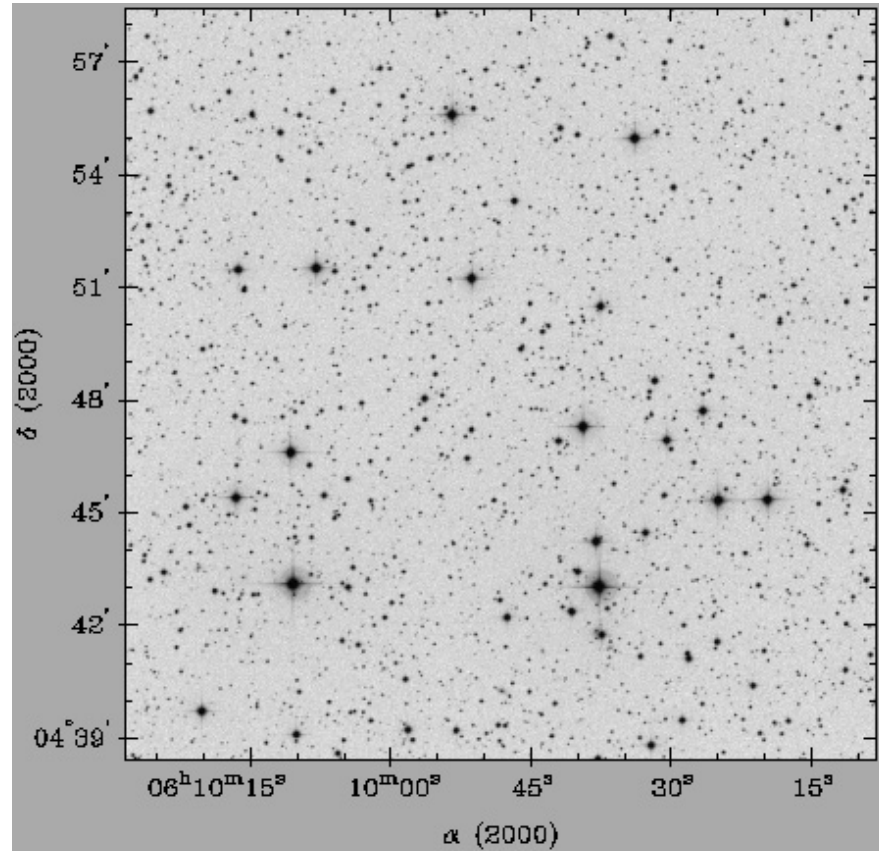

Fig. 1. $20^{\prime} \times 20^{\prime}\left(\approx 5.3 \times 5.3 \mathrm{pc}^{2}\right)$ XDSS $R$ image of NGC 2180 .

http://obswww.unige.ch/webda is the object's designation.

The original coordinates of NGC 2180 precessed to $J 2000$ are $\alpha=06^{\mathrm{h}} 09^{\mathrm{m}} 36^{\mathrm{s}}$ and $\delta=+04^{\circ} 43^{\prime}$. However, in what follows we revised the central coordinates to $\alpha=06^{\mathrm{h}} 09^{\mathrm{m}} 48^{\mathrm{s}}$ and $\delta=+04^{\circ} 48^{\prime} 26^{\prime \prime}$ based on bright star membership (Sect. 3). The latter coordinates convert to $\ell=203.85^{\circ}$ and $b=-7.01^{\circ}$. A Digitized Sky Survey (XDSS) $R$ image of NGC 2180, centered on the revised coordinates is given in Fig. 1.

Visually, the cluster is sparse and poorly populated, but a few bright stars are present in the area.

\subsection{The dynamically evolved open cluster NGC 3680}

The intermediate-age open cluster NGC 3680 has already been extensively studied. Its slightly supersolar metallicity was determined by Eggen (1969) and through the photoelectric $u v b y$ $\mathrm{H}_{\beta}$ photometry of Nissen (1988). CCD uvby photometry of the central parts of NGC 3680 was published by Anthony-Twarog et al. (1989), and CCD and photographic $B V$ photometry by Anthony-Twarog et al. (1991).

Previous age estimates for NGC 3680 varied from $\tau_{\text {age }} \sim$ 1.0 Gyr (Mazzei \& Pigatto 1988) to $\tau_{\text {age }} \sim 4.5$ Gyr (AnthonyTwarog et al. 1991). The Strömgren photometry of Bruntt et al. (1999) resulted in an age of $\tau_{\text {age }}=1.45 \pm 0.15 \mathrm{Gyr}$, comparable to the value $\tau_{\text {age }}=1.6 \pm 0.5 \mathrm{Gyr}$ derived by Kozhurina-Platais et al. (1997).

With respect to the dynamical state, Nordström et al. (1996), based on by CCD photometry of 310 stars as well as radial velocity and proper motion data, concluded that NGC 3680 should be the last remnant of a cluster in an advanced state of dissolution, almost lost in the foreground field of similar stars. They estimate the present stellar mass as $m_{\mathrm{obs}} \sim 100 M_{\odot}$, and an initial total mass of $\sim 1200 M_{\odot}$. 


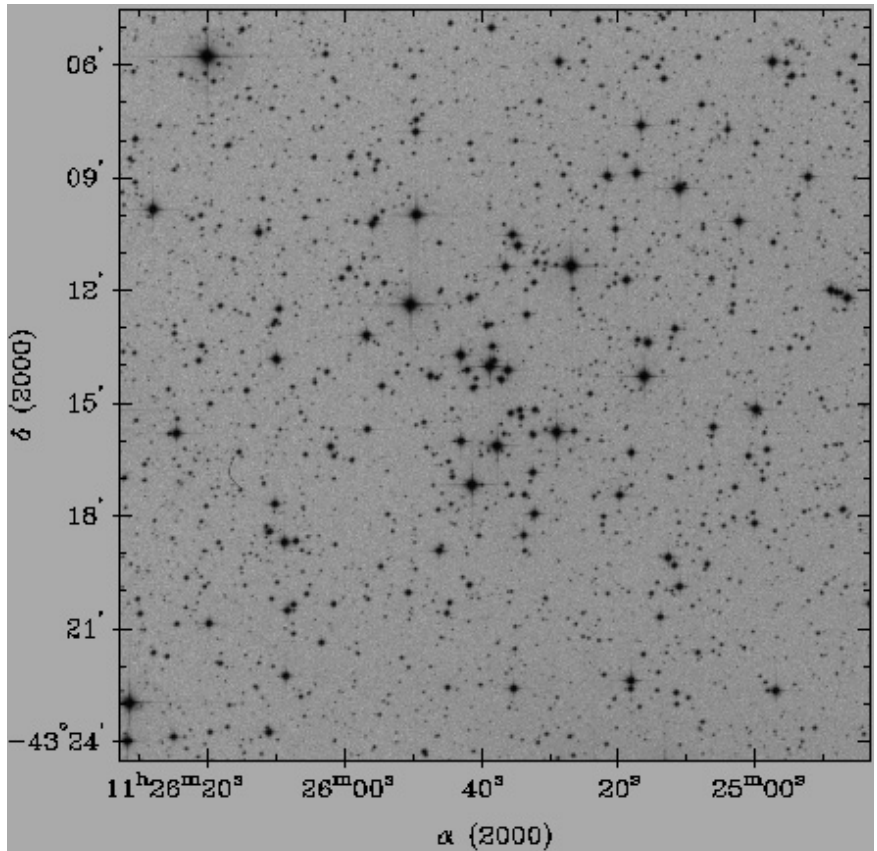

Fig. 2. $20^{\prime} \times 20^{\prime}\left(\approx 5.8 \times 5.8 \mathrm{pc}^{2}\right)$ XDSS $R$ image of NGC 3680 .

A Digitized Sky Survey (XDSS) $R$ image of NGC 3680 is given in Fig. 2, in which a concentration of bright stars is present in the central $8^{\prime} \times 8^{\prime}$. The central coordinates of this intermediate-age open cluster, estimated from the XDSS image, are $\alpha=11^{\mathrm{h}} 25^{\mathrm{m}} 38^{\mathrm{s}}$ and $\delta=-43^{\circ} 14^{\prime} 30^{\prime \prime}$. These values agree with the WEBDA coordinates. The new coordinates convert to $\ell=286.76^{\circ}$ and $b=16.92^{\circ}$.

\section{The 2MASS photometry}

We investigate the nature and structure of both clusters using $J$ and $H$ photometry obtained from the 2MASS All Sky data release, which is available at http://www . ipac . caltech. edu/2mass/releases/allsky/. 2MASS photometric errors typically attain 0.10 mag at $J \approx 16.2$ and $H \approx 15.0$, see e.g. Soares \& Bica (2002). Star extractions have been performed using the VizieR tool at http://vizier.u-strasbg.fr/ viz-bin/VizieR?-source=2MASS. For each cluster we made circular extractions centered on the coordinates given in Sect. 2. In order to maximize the statistical significance and representativity of background star counts, we decided to use an external ring (same area as the cluster) as offset field. This offset field will be used to represent the stellar background contribution to the cluster. We used an extraction radius of $40^{\prime}$ for NGC 2180 and 30' for NGC 3680.

In Fig. 3 we show the $J \times(J-H)$ CMDs for each cluster (left panels) along with the corresponding (same area) offset fields (right panels). In order to maximize the cluster/background contrast, the CMDs have been built with stars extracted within $10^{\prime}$ and $15^{\prime}$, respectively for NGC 2180 and NGC 3680. According to the analysis in Sect. 4, these dimensions are smaller than the limiting radius of each cluster.

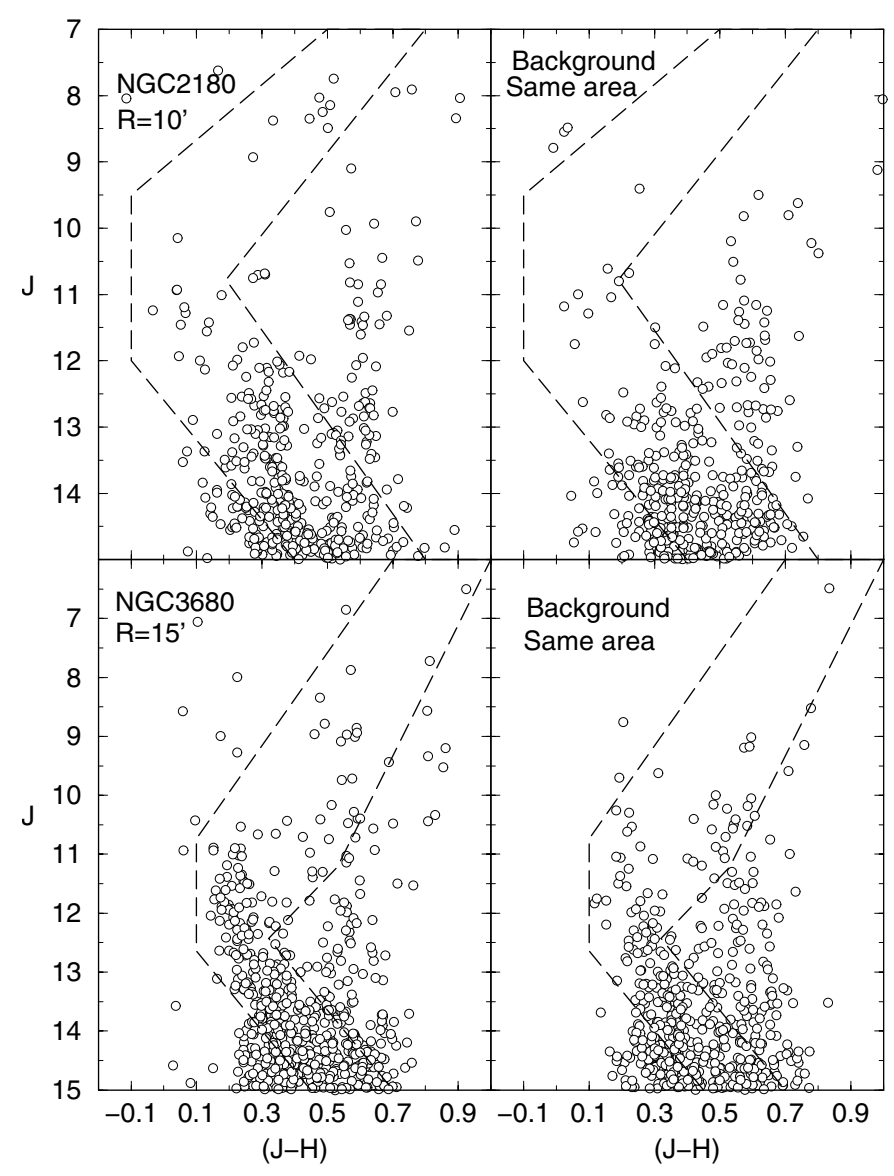

Fig. 3. $J \times(J-H)$ CMDs for the cluster NGC 2180 and corresponding offset field (top panels), and for NGC 3680 (bottom panels). Colour filters are shown by the dashed lines.

NGC 2180 may be recognized as a cluster by the presence of the MS and a group of bright giants. These features are not present in its comparison field (top-right panel). Since the 6 giant stars are located within the central $10^{\prime}$, we recalculated the central coordinates of NGC 2180 as the average values for these stars (Sect. 2).

The MS in the CMD of NGC 3680 is well-defined, particularly near the turnoff, and the presence of giants is also clear.

In all CMDs shown in Fig. 3, the contribution of disk stars is seen as nearly vertical sequences at $(J-H) \approx 0.6$ and $(J-H) \approx 0.35$. These contributions, as well as that of faint and spurious detections, have to be properly taken into account in order to isolate the cluster members. Indeed, it is interesting to note that the number of sources in the CMD in the direction of NGC 2180 is 1410, while in the CMD of its comparison field, this number is 1399 . For NGC 3680, the above numbers are 1887 and 1890, respectively for cluster and comparison field.

\section{Cluster structure}

The overall cluster structure is analysed by means of the star density radial distribution, defined as the number of stars per area in the direction of a cluster, which is shown in Fig. 4 for NGC 2180 (top panel) and NGC 3680 (bottom panel). 


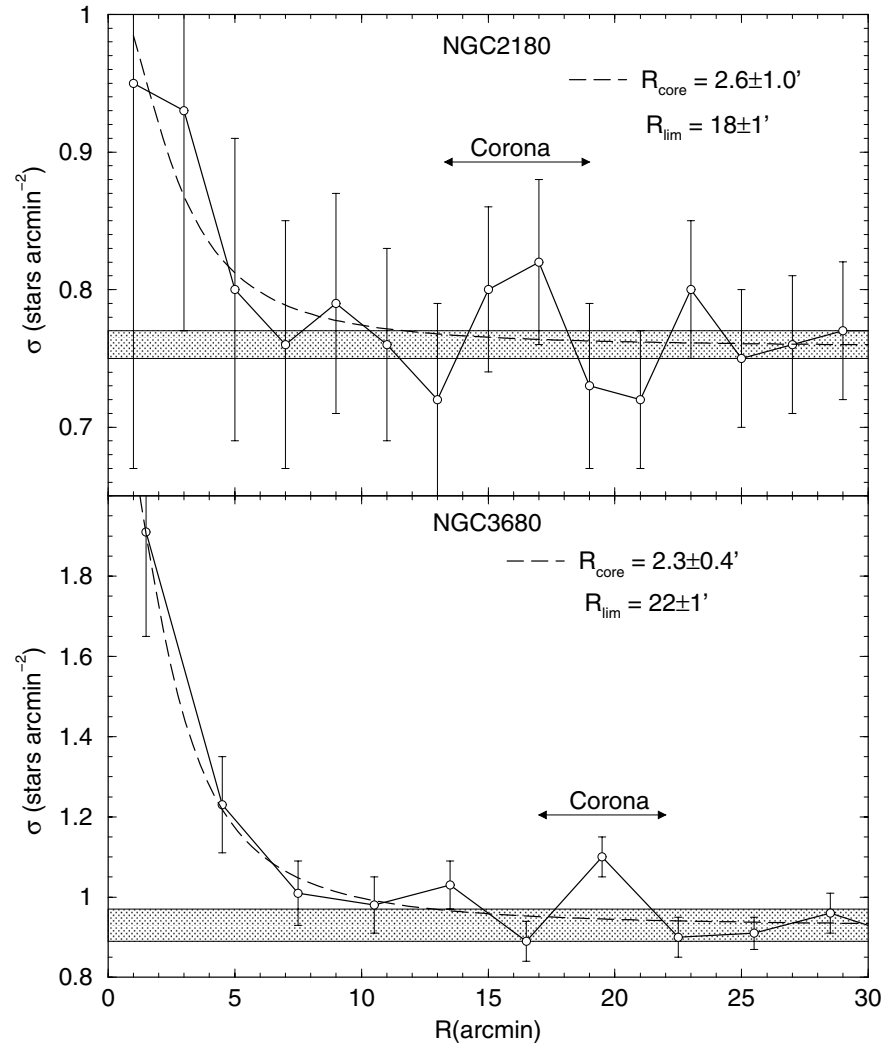

Fig. 4. Radial distribution of surface star density. The average background levels are shown as shaded rectangles; $1-\sigma$ Poisson errors are also shown. For both clusters, magnitude $(J<15.0)$ and colour cutoffs have been applied to the object and offset fields. The dashed lines show a surface density profile (King law) fit to the radial distribution of stars; the resulting core radius for each cluster is indicated.

Before counting stars, we applied a cutoff $(J<15.0)$ to both clusters and corresponding offset fields to avoid oversampling, i.e. to avoid spatial variations in the number of faint stars which are numerous, affected by large errors, and may include spurious detections. Colour filters (dashed lines in Fig. 3) in the CMDs have also been applied to both clusters and corresponding offset fields, in order to account for the contamination of the Galaxy. This procedure has been applied in the analysis of the open cluster M 67 (Bonatto \& Bica 2003). As a result of the filtering process, the number of stars in the direction of NGC 2180 turns out to be 253 , compared to 238 in its offset field. For NGC 3680, these numbers are 755 and 668, respectively for cluster and comparison field. The radial distributions have been determined by counting stars inside concentric annuli with a step of $2.0^{\prime}$ in radius for NGC 2180 and $2.5^{\prime}$ for NGC 3680, and dividing the number of stars by the respective annulus area. The background contribution level, shown in Fig. 4 as shaded rectangles, corresponds to the average number of stars present in the external annuli.

The radial density profile of NGC 2180 (top panel) is not smooth, presenting bumps and dips with respect to the background level. The relatively small number of stars in this cluster is reflected by the large $1-\sigma$ Poisson error bars. Even so, NGC 2180 appears to have a central concentration of stars for $R<5^{\prime}$ as well as an excess in the corona at $14^{\prime} \leq R \leq 18^{\prime}$.
Considering the profile fluctuations with respect to the background level, most of the cluster's stars can be considered to be contained within a radius of $\approx 10^{\prime}$. However, a corona (Sect. 5) is detected, and we adopt as limiting radius $R_{\text {lim }} \approx 18^{\prime}$. The limiting radius corresponds to the radius at which the cluster's profile merges into the background level (Fig. 4). On the other hand, the radial density profile of NGC 3680 (bottom panel) is smooth and presents a well-defined central concentration of stars, as well as an excess in the corona at $17^{\prime} \leq R \leq 22^{\prime}$. Its limiting radius lies at $R_{\lim } \approx 22^{\prime}$.

Although the spatial shape of the clusters may not be perfectly spherical, the King law (1966) is still useful to derive first order structural parameters. A cluster core radius $R_{\text {core }}$ can be calculated by fitting a King surface density profile $\sigma(R)=\frac{\sigma_{0}}{1+\left(R / R_{\text {core }}\right)^{2}}$ to the background-subtracted radial distribution of stars. The fits have been performed using a non-linear least-squares fit routine which uses the error bars as weights. The resulting fits are shown in Fig. 4, as dashed lines. The radial density profile of NGC 3680 (bottom panel) follows well King law, with a resulting core radius $R_{\text {core }}=2.3 \pm 0.4^{\prime}$. The large $1-\sigma$ Poisson errors and non-uniform density profile of NGC 2180 produce a significant uncertainty in the resulting core radius, $R_{\text {core }}=2.6 \pm 1.0^{\prime}$. Using the cluster distances derived in Sect. 5 below, the linear core radii turn out to be $R_{\text {core }}=0.7 \pm 0.3 \mathrm{pc}$ and $R_{\text {core }}=0.7 \pm 0.1 \mathrm{pc}$, respectively for NGC 2180 and NGC 3680 . Finally, the angular diameters $(2 \times$ $\left.R_{\lim }\right)$ of $36 \pm 2^{\prime}$ and $44 \pm 2^{\prime}$, convert to linear limiting diameters of $9.5 \pm 1.2 \mathrm{pc}$ and $12.8 \pm 1.3 \mathrm{pc}$, respectively for NGC 2180 and NGC 3680.

\section{Fundamental parameters}

To maximize cluster membership probability, the analyses in the following two sections will be restricted to stars extracted within $R_{\lim }$ (Sect. 4). Cluster parameters will be derived using solar metallicity Padova isochrones from Girardi et al. (2002) computed with the 2MASS $J, H$ and $K_{\mathrm{S}}$ filters (available at http://pleiadi.pd.astro.it). The solar metallicity isochrones have been selected to be consistent with the results of Eggen (1969) and Nissen (1988), at least for NGC 3680. The 2MASS transmission filters produced isochrones very similar to the Johnson ones, with differences of at most 0.01 in $(J-H)$ (Bonatto et al. 2004). For reddening and absorption transformations we use $R_{V}=3.2$, and the relations $A_{J}=0.276 \times A_{\mathrm{V}}$ and $E(J-H)=0.33 \times E(B-V)$, according to Dutra et al. (2002) and references therein.

We show in Fig. 5 the isochrone fits to the $M_{J} \times(J-H)$ CMD of NGC 2180 (left panel) and NGC 3680 (right panel). $M_{J}$ values are obtained after applying the distance modulus derived below for each cluster.

The upper MS of NGC 2180 is relatively depleted of stars, with a single star near the turnoff at $M_{J} \approx 0.0$. Thus, the solar-metallicity fit which best matches the MS features has been obtained with the $710 \mathrm{Myr}$ isochrone. This solution is shown as a solid line in Fig. 5. The isochrone fit and related uncertainties result in a distance modulus $(m-M)_{0}=$ $10.10 \pm 0.20, E(B-V)=0.0$ and a distance to the Sun $d_{\odot}=$ $1.05 \pm 0.08 \mathrm{kpc}$. However, the above isochrone solution fails to 


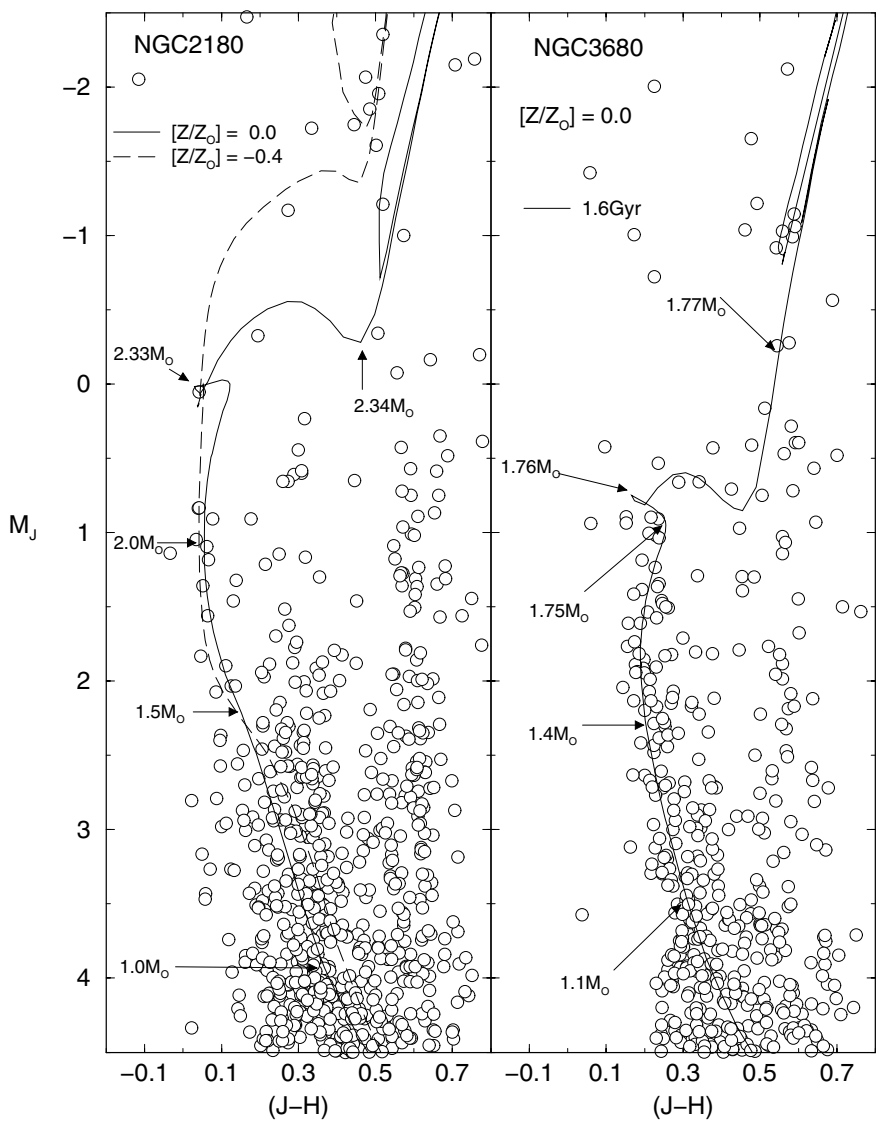

Fig. 5. Isochrone fits to the $M_{J} \times(J-H)$ CMDs of NGC 2180 (left panel) and NGC 3680 (right panel). For NGC 2180, equally good fits are obtained with the $710 \mathrm{Myr}$ isochrones with metallicity $\left[Z / Z_{\odot}\right]=$ 0.0 (solid line) and $\left[Z / Z_{\odot}\right]=-0.4$ (dashed line). For the $\left[Z / Z_{\odot}\right]=0.0$ solution, $(m-M)_{0}=10.10 \pm 0.20, E(B-V)=0.0$ and $d_{\odot}=1.05 \pm$ $0.08 \mathrm{kpc}$, while the $\left[Z / Z_{\odot}\right]=-0.4$ solution results in $(m-M)_{0}=$ $9.40 \pm 0.20, E(B-V)=0.18$ and $d_{\odot}=0.76 \pm 0.06 \mathrm{kpc}$. For NGC 3680 we derive an age of $\tau_{\text {age }} \sim 1.6 \mathrm{Gyr},(m-M)_{0}=10.00 \pm$ $0.20, E(B-V)=0.00$ and $d_{\odot}=1.00 \pm 0.09 \mathrm{kpc}$. Representative stellar masses are indicated for both clusters.

reproduce the 6 giants at $M_{J} \approx-2$ and $(J-H) \approx 0.5$, which might be accounted for by differences in metallicity. Accordingly, we present an alternative fit with the subsolar metallicity $\left(\left[Z / Z_{\odot}\right]=-0.38\right), 710 \mathrm{Myr}$ isochrone, which results in a distance modulus $(m-M)_{0}=9.40 \pm 0.20, E(B-V)=$ 0.18 and $d_{\odot}=0.76 \pm 0.06 \mathrm{kpc}$. According to this solution (dashed line), the 6 bright stars would be, in fact, the giant clump of NGC 2180. The giant clump in NGC 2180 is similar to that of the Hyades cluster (e.g. Perryman et al. 1998). Both clusters have similar ages. Since the CMD features below the turnoff are equally well reproduced by both isochrones, the intrinsic metallicity of NGC 2180 is probably in the range $-0.4 \leq\left[Z / Z_{\odot}\right] \leq 0.0$. In the same way, we adopt as distance to the Sun the average of the values derived for both isochrones, i.e. $d_{\odot}=0.91 \pm 0.15 \mathrm{kpc}$. With the above distance to the Sun, the galactocentric distance of NGC 2180 becomes $8.8 \pm$ $0.1 \mathrm{kpc}$. In the subsequent calculations we will adopt the solar metallicity solution as reference, particularly to derive the LFs and MFs.
The presence of a well-defined turnoff and giant stars in the CMD of NGC 3680 constrain the isochrone fits to the $\tau_{\text {age }}=1.6 \mathrm{Gyr}$ solution. For this cluster we derive $(m-M)_{0}=$ $10.00 \pm 0.20, E(B-V)=0.00$ and $d_{\odot}=1.00 \pm 0.09 \mathrm{kpc}$. The galactocentric distance of NGC 3680 is $7.8 \pm 0.1 \mathrm{kpc}$. The present age estimate, using 2MASS photometry, is in close agreement with those derived by Kozhurina-Platais et al. (1997) and Bruntt et al. (1999).

\section{Luminosity and mass functions}

In this section we analyze the observed star counts as a function of magnitude (LF) and mass (MF) as well as their spatial dependence.

The accurate determination of a cluster's LF (or MF) suffers from some problems, in particular $(i)$ the contamination of cluster members by field stars; (ii) the observed incompleteness at low-luminosity (or low-mass) stars; and (iii) the mass segregation, which may affect even poorly populated, relatively young clusters (Scalo 1998). The 2MASS uniform sky coverage allows one to overcome, at least in part, points $(i)$ - since suitable offset fields can be selected around the cluster and (iii) the entire cluster area can be included in the analyses. Thus, advanced stages of mass segregation would affect more significantly the analysis of very old, dynamically evolved clusters (e.g. M 67, Bonatto \& Bica 2003).

In Fig. 6 we show the LFs $\left(\phi\left(M_{J}\right)\right)$ in the $J$ filter (shaded area) for the two clusters, built as the difference of the number of stars in a given magnitude bin between object (continuous line) and average offset field (dotted line). The LFs are given in terms of the absolute magnitude $M_{J}$, obtained after applying the distance modulus derived in Sect. 5 for each cluster, and the bin in magnitude used is $\Delta M_{J}=0.50 \mathrm{mag}$. We remind that the LFs in Fig. 6 are built after applying magnitude $(J<15.0)$ and colour cutoffs to the objects and their offset fields (Sect. 5). The LFs for different spatial regions of NGC 2180 are in the left panels, and those of NGC 3680 are in the right panels.

To search for spatial variations in the stellar content, we built LFs for different regions in and around the clusters, according to the structures present in the radial density profiles (Fig. 4). Thus, the first LF encompasses the central region of each cluster (panels d), $0.0^{\prime} \leq R \leq 5.0^{\prime}$ and $0.0^{\prime} \leq R \leq 7.5^{\prime}$, respectively for NGC 2180 and NGC 3680 ; the second one corresponds to the outskirts (panels c), $5.0^{\prime} \leq R \leq 10.0^{\prime}$ and $7.5^{\prime} \leq$ $R \leq 15.0^{\prime}$, and the third LF to the corona (panels b). In panels (a) we show the overall LFs $\left(0.0^{\prime} \leq R \leq R_{\text {lim }}\right)$. The background $\phi\left(M_{J}\right)$ has been scaled to match the projected area of each region. Representative MS spectral types (adapted from Binney \& Merrifield 1998) are shown in the top panels. The turnoff is indicated in all panels. Giants are contained within $10^{\prime}$ in NGC 2180 and $15^{\prime}$ in NGC 3680.

The severe depletion of MS stars in NGC 2180 can be clearly seen in the internal background-subtracted LFs (Fig. 6, left panels $\mathrm{c}$ and $\mathrm{d}$ ) which, in fact, are similar to each other. The above spatial properties of the LFs probably reflect mass segregation followed by advanced and significant Galactic tidal stripping effects (severe depletion of stars in the corona). Indeed, the overall cluster and offset field LFs (left panel a) 

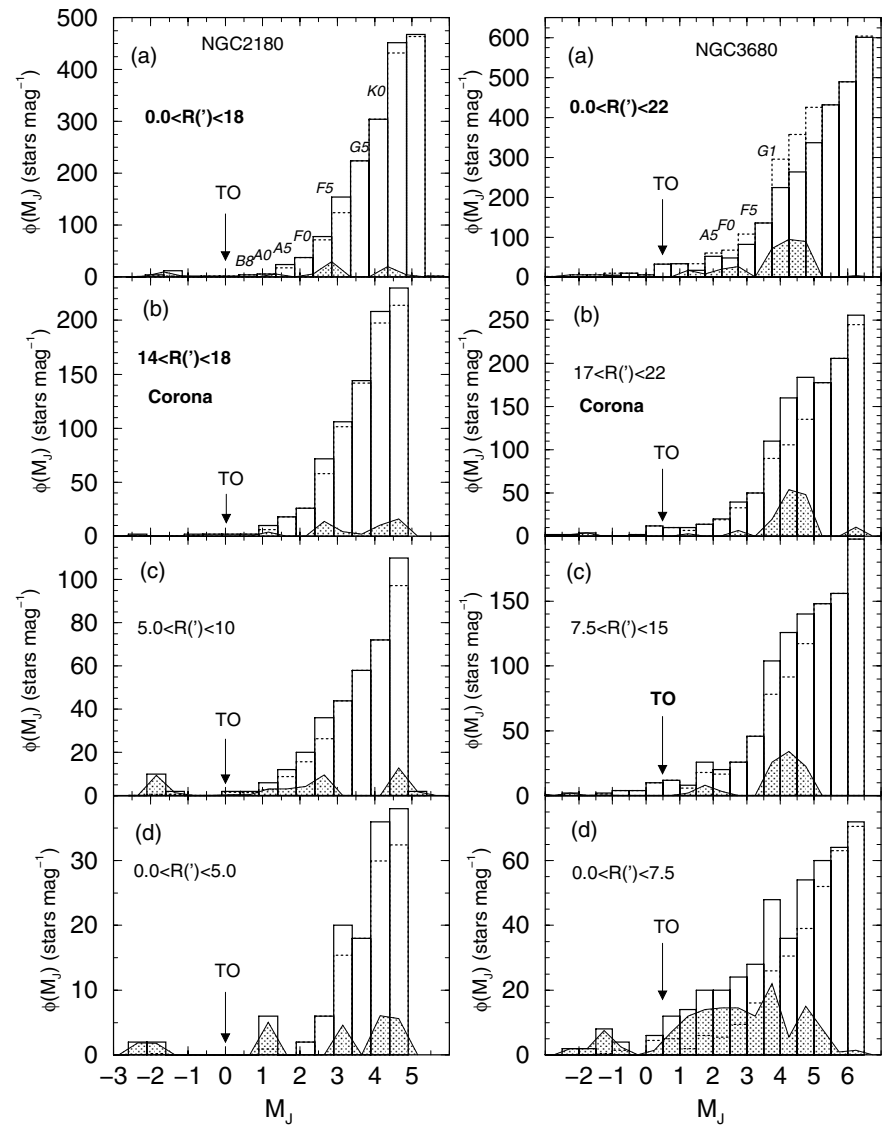

Fig. 6. Luminosity functions $\left(\phi\left(M_{J}\right)\right)$ in terms of the absolute magnitude $M_{J}$. Magnitude $(J<15.0)$ and colour cutoffs have been applied to the object and offset fields. Continuous line: star counts in the cluster's area; dotted line: star counts in the offset field; shaded area: background-subtracted LF. The turnoff (TO) is indicated in each panel.

are very similar, indicating that the original stellar content of NGC 2180 is already nearly dispersed into the background population. In order to reach such an advanced dynamical state in a time span of $\sim 700 \mathrm{Myr}$ it is reasonable to assume that the initial cluster was not massive.

On the other hand, the central LF of NGC 3680 (Fig. 6, right panel d) is nearly flat from the turnoff (spectral type $\sim \mathrm{A} 0)$ to the turnover $(\sim \mathrm{G} 1)$, confirming the central depletion of lower-MS stars found by Anthony-Twarog et al. (1997). This effect may be accounted for by mass segregation alone. Indeed, the $7.5^{\prime} \leq R \leq 15^{\prime}$ and corona regions (right panels $\mathrm{c}$ and $\mathrm{b}$ ) are still well populated by low-mass $(\sim \mathrm{G} 0)$ stars, suggesting that the Galactic tidal stripping has not yet been effective in severely depleting this cluster. In this sense, NGC 3680 is very similar to the $\tau_{\text {age }} \approx 3.2 \mathrm{Gyr}$, mass-segregated open cluster M 67, as spatially analysed in Bonatto \& Bica (2003).

The overall LFs in Fig. 6 (top panels), restricted to the region between the turnoff and turnover, have been converted to MFs according to $\phi(m)=\phi\left(M_{J}\right)\left|\frac{\mathrm{d} m}{\mathrm{~d} M_{J}}\right|^{-1}$. We used the stellar mass-luminosity relations taken from the Padova isochrones (Sect. 5), 710 Myr for NGC 2180 and 1.6 Gyr for NGC 3680. Since we are interested in obtaining only an estimate of the mass in NGC 2180, we used the solar metallicity isochrone to

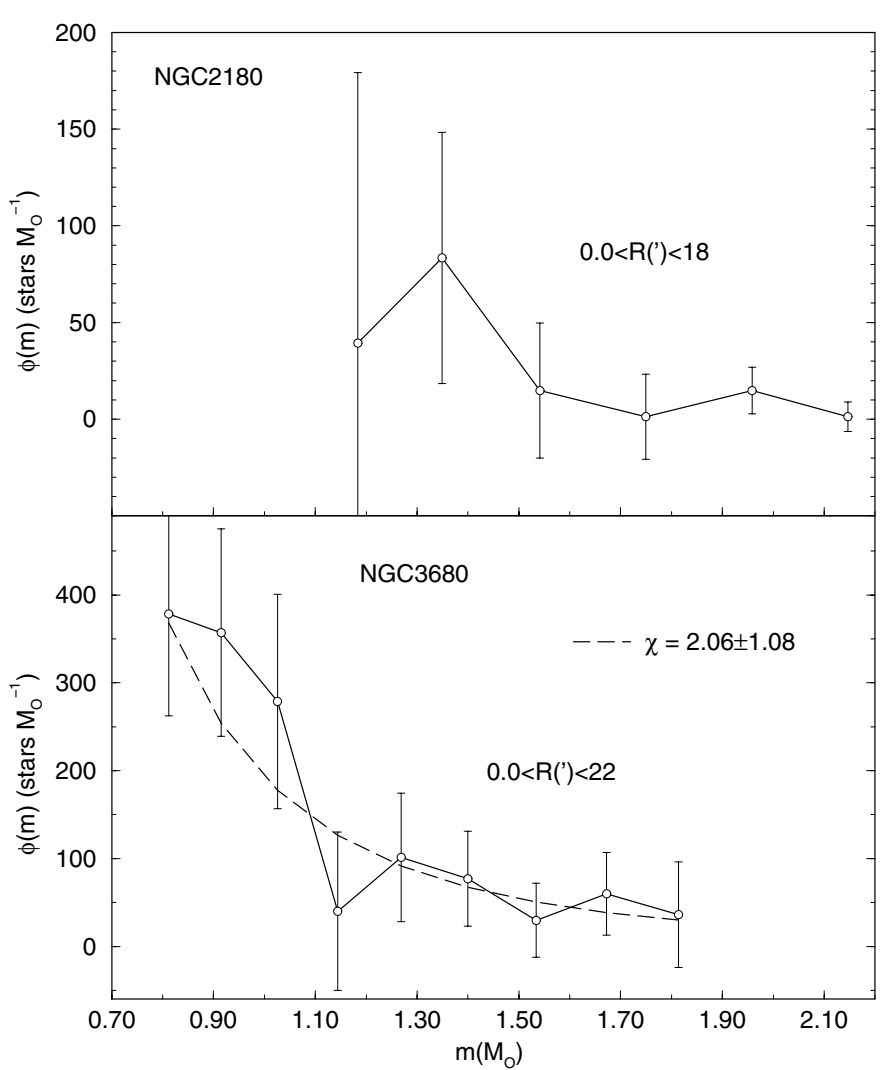

Fig. 7. Turnoff-turnover mass functions. The MF fit $\phi(m) \propto m^{-(1+\chi)}$ for NGC 3680 is shown as a dashed line.

derive the mass-luminosity relation. The resulting MFs, including $1-\sigma$ error bars, are shown in Fig. 7.

Despite the non-uniform overall MF of NGC 3680, we applied a fit of $\phi(m) \propto m^{-(1+\chi)}$ to the turnoff $\left(\approx 1.8 M_{\odot}, \approx \mathrm{A} 0\right)-$ turnover $\left(\approx 1.0 M_{\odot}, \approx \mathrm{G} 1\right)$ region, which resulted in a slope $\chi=2.06 \pm 1.08$, a steeper MS than a Salpeter's $\chi=1.35$. Numerical integration of the MF in the above mass interval, taking into account the uncertainties, resulted in a MS mass of $m_{\mathrm{MS}}=111 \pm 24 M_{\odot}$. We estimate the mass stored in the giants $\left(m_{\text {giant }}\right)$ by counting the number of stars brighter than the turnoff present in the overall LFs, and multiplying this number by the mass at the turnoff, resulting in $m_{\text {giant }} \approx 19 M_{\odot}$ for NGC 3680 . Thus, the present observed stellar mass in NGC 3680 is $m_{\mathrm{obs}}=$ $130 \pm 24 M_{\odot}$.

Assuming that the very low-mass stars have not yet been stripped away from the cluster, the total mass locked up in stars can be estimated by directly extrapolating the present MF fit down to the theoretical stellar low-mass end $m_{\text {low }}=0.08 M_{\odot}$. The resulting total stellar mass in NGC 3680 is $\sim(2.4 \pm 1.2) \times$ $10^{3} M_{\odot}$ which, within errors, is similar to the value found by Nordström et al. (1996). This agreement is interesting, considering the different methods of taking into account the stellar background. On the other hand, Kroupa et al. (1991) and Kroupa (2001) presented evidence that the MFs of most globular and open clusters flatten below $\sim 0.5 M_{\odot}$. As a consequence, the total stellar mass in NGC 3680 would be less than the value derived above, since most of the stars in a cluster are expected to be found in the low-mass range. Accordingly, we derive a more conservative total mass value for NGC 3680 assuming the 
Table 1. Open cluster and remnant parameters.

\begin{tabular}{lccccccc}
\hline \hline Cluster & $\begin{array}{c}\ell \\
\left({ }^{\circ}\right)\end{array}$ & $\begin{array}{c}b \\
\left.{ }^{\circ}\right)\end{array}$ & $\begin{array}{c}d_{\mathrm{GC}} \\
(\mathrm{kpc})\end{array}$ & $\begin{array}{c}\text { Age } \\
(\mathrm{Gyr})\end{array}$ & $\begin{array}{c}R_{\text {core }} \\
(\mathrm{pc})\end{array}$ & $\begin{array}{c}R_{\lim } \\
(\mathrm{pc})\end{array}$ & $\begin{array}{c}m_{\mathrm{obs}} \\
\left(M_{\odot}\right)\end{array}$ \\
\hline NGC 2180 & 203.85 & -7.01 & $9.0^{a}$ & $0.7 \pm 0.1^{a}$ & $0.7 \pm 0.3^{a}$ & $4.7 \pm 0.6^{a}$ & $47 \pm 7^{a}$ \\
NGC 3680 & 286.76 & 16.92 & $8.8^{a}$ & $1.6 \pm 0.1^{a}$ & $0.7 \pm 0.1^{a}$ & $6.4 \pm 0.6^{a}$ & $130 \pm 24^{a}$ \\
M 67 & 215.68 & 31.93 & $8.6^{b}$ & $3.2 \pm 0.1^{b}$ & $1.2 \pm 0.1^{b}$ & $8.7 \pm 0.8^{b}$ & $344 \pm 40^{b}$ \\
NGC 188 & 122.85 & 22.39 & $8.9^{c}$ & $7.1 \pm 0.1^{c}$ & $1.3 \pm 0.1^{c}$ & $9.7 \pm 0.4^{c}$ & $380 \pm 12^{c}$ \\
NGC 1252 & 274.08 & -50.83 & $7.8^{d}$ & $3.0 \pm 1.0^{d}$ & - & $0.7 \pm 0.1^{a}$ & $10 \pm 5^{a}$ \\
NGC 7772 & 102.73 & -44.27 & $8.4^{d}$ & $1.5 \pm 0.5^{e}$ & - & $0.9 \pm 0.1^{g}$ & $10 \pm 5^{a}$ \\
NGC 7036 & 64.54 & -21.44 & $7.6^{d}$ & $3.5 \pm 0.5^{e}$ & - & $1.0 \pm 0.1^{g}$ & $10 \pm 5^{a}$ \\
Ruprecht 3 & 238.77 & -14.81 & $8.4^{d}$ & $1.5 \pm 0.5^{f}$ & - & $0.2 \pm 0.0^{a}$ & $10 \pm 5^{a}$ \\
\hline
\end{tabular}

Notes. Column (4): Galactocentric distance; Data sources are: ${ }^{a}$ this paper, using 2MASS; ${ }^{b}$ Bonatto \& Bica (2003); ${ }^{c}$ Bonatto et al. (2004); ${ }^{d}$ Pavani et al. (2002); ${ }^{e}$ Carraro (2002); ${ }^{f}$ Pavani et al. (2003); ${ }^{g}$ this paper, using Carraro (2002).

universal IMF of Kroupa (2001), in which $\chi=0.3 \pm 0.5$ for $0.08 M_{\odot}-0.50 M_{\odot}$, and $\chi=1.3 \pm 0.3$ for $0.50 M_{\odot}-0.80 M_{\odot}$. For $0.80 M_{\odot}-1.80 M_{\odot}$ we adopt the value derived in this paper, $\chi=2.06 \pm 1.08$. As expected, the resulting total stellar mass decreases to $546 \pm 206 M_{\odot}$.

Similar to NGC 3680, we derive a giant mass of $m_{\text {giant }} \approx$ $16 M_{\odot}$ for NGC 2180 . However, the considerable variations in the MF do not allow a statistically significant fit. Consequently, the present observed stellar mass in NGC 2180 has been estimated by numerically integrating its MF (Fig. 7, top panel) and adding $m_{\text {giant }}$ to this value. The resulting observed stellar mass is $m_{\mathrm{obs}} \sim 47 \pm 7 M_{\odot}$. Considering a severe low-mass star depletion (Bergond et al. 2001; de la Fuente Marcos 1996) for NGC 2180, it would certainly be less massive than NGC 3680, which is older and at a somewhat smaller Galactocentric distance (Sect. 7).

Taking into account the mass estimates, the derived ages of both clusters are more than an order of magnitude larger than the corresponding $t_{\text {relax }}$, which is consistent with the significant presence of mass-segregation effects.

\section{Comparison with other dynamical states}

At this point, it may be useful to see where NGC 2180 and NGC 3680 fit in the context of $(i)$ well-known dynamically evolved open clusters; and (ii) poorly populated remnants of open clusters. In particular, we will pay attention to parameters intimately associated with dynamical evolution, i.e., age, core and limiting radii and stellar mass. The remnants are NGC 1252 (Pavani et al. 2002), Ruprecht 3 (Pavani et al. 2003), NGC 7036 and NGC 7772 (Carraro 2002). The old open clusters are M 67 (Bonatto \& Bica 2003) and NGC 188 (Bonatto et al. 2004), both more massive than NGC 2180 and NGC 3680. All these objects have been analysed by means of 2MASS photometry following the same methods as in the case of NGC 2180 and NGC 3680, thus ensuring homogeneity in terms of analysis and derived parameters. Relevant parameters for the objects are given in Table 1. The results are shown in Fig. 8. Part of the parameters for NGC 1252, Ruprecht 3, NGC 7036 and NGC 7772 have been derived using 2MASS data, following a similar analysis as that used for NGC 2180 and NGC 3680.

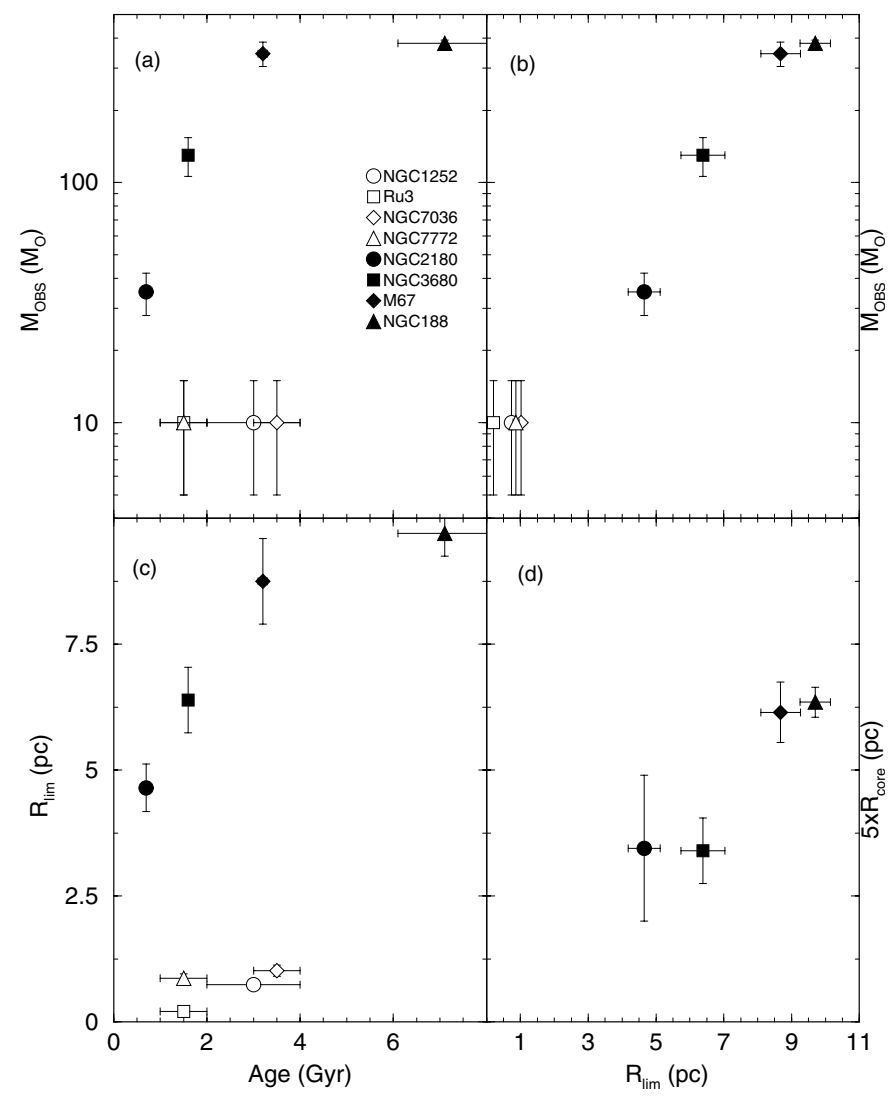

Fig. 8. NGC 2180 and NGC 3680 compared to dynamically evolved open clusters and known open cluster remnants. For visualization purposes, the ordinate in panel d) corresponds to $5 \times R_{\text {core }}$.

As expected, the structural parameters of the intrinsic open clusters present a strong dependence with time. This is particularly true for the limiting radius (panel c), which increases linearly with time, probably as a consequence of the Galactic tidal pull. The observed mass, on the other hand, initially increases linearly with time and then reaches a saturation threshold (panel a). This threshold is defined by M 67 and NGC 188, both dynamically advanced open clusters, more massive than NGC 2180 and NGC 3680, with strong effects of mass segregation. The relations of the core radius to the limiting radius (panel d), and of $R_{\text {lim }}$ to $m_{\mathrm{obs}}$ (panel b), present 
a similar pattern, linear increase first followed by a saturation. This behaviour may be accounted for by a combination of the Galactic tidal pull (increasing $R_{\lim }$ with time) and mass segregation (initial core formation and growth, followed by core stabilization (de la Fuente Marcos 1996)). In the far future, these massive open clusters will leave behind only a core, with most of the low-mass stars dispersed into the background (de la Fuente Marcos 1996).

With respect to the structural parameters, the open clusters seem to follow a well-defined sequence (panels a-d). The remnants, however, follow this sequence only for $R_{\text {lim }} \times m_{\mathrm{obs}}$ (panel b), since most of their stellar content may have already been dispersed into the background.

NGC 2180 is consistently closer than NGC 3680 to the loci occupied by the remnants in panels (a), (b) and (c). Despite being younger than NGC 3680, the less-massive nature of NGC 2180 probably accelerated its evolutionary time-scale, setting it in a more dynamically advanced state than NGC 3680.

Interestingly, the remnants dealt with in this paper have Galactocentric distances smaller that those of the evolved open clusters (Col. 4 of Table 1). Again, this suggests the effects of the Galactic tidal pull (external evolution). However, to draw objective conclusions with respect to open cluster evolution it is necessary to take separately into account selection and intrinsic evolution effects. To further explore these issues we intend to analyze a larger sample of clusters which span a variety of photometric, structural and dynamical properties, using the same methods employed in the present paper.

\section{Proper motion in the direction of both objects}

Open clusters are known to contain significant fractions of binary stars, and dynamical evolution tends to enhance this fraction (e.g. Montgomery et al. 1993; von Hippel \& Sarajedini 1998). One effect of a significant fraction of binaries in a cluster is to produce large deviations in the cluster's proper motion distribution with respect to a single-star component, since the resulting velocities may be much larger for the binary motions. In this sense, proper motion determinations should not be taken at face-value to characterize (or rule out) the physical nature of a star cluster, particularly for dynamically advanced clusters (which is the case of NGC 3680 and possibly NGC 2180). Proper motions for both NGC 3680 and NGC 2180 fields have been obtained from $\mathrm{UCAC}^{1}$.

In Fig. 9 we show the proper motion (modulus) distribution in the central regions of NGC 3680 and NGC 2180. The offset-field stars contribution to each object (scaled to match the projected area of the central region) is shown as a shaded curve. In NGC 3680 a peak clearly appears in the distribution which corresponds to the single-star component. However, besides the single-star component, both the offset field and cluster present stars with large proper motions, which can be accounted for by binary motions. A similar effect, although with a smaller number of stars, can be observed in the central region of NGC 2180. From this figure a few binaries (larger proper motions) can be inferred. Most of the single stars

\footnotetext{
1 The Second US Naval Observatory CCD Astrograph Catalog (Zacharias et al. 2003).
}

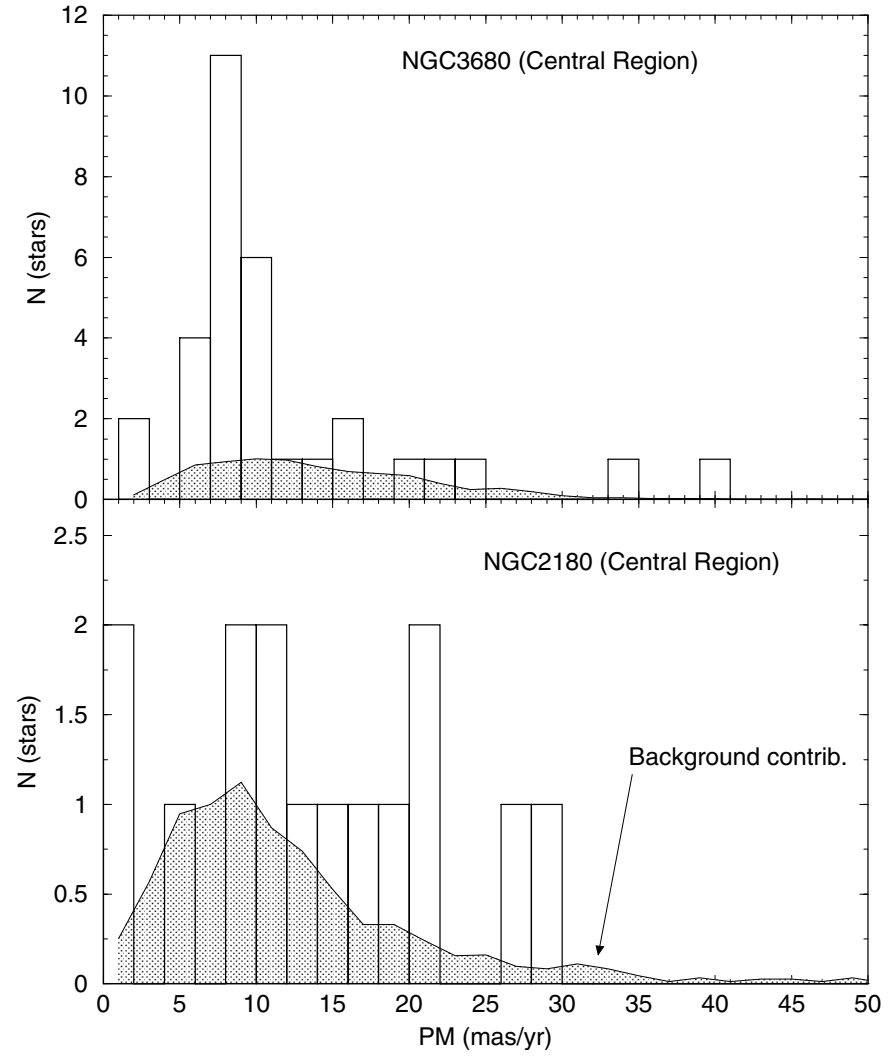

Fig. 9. Proper motion distribution in the central regions of NGC 2180 and NGC 3680 (solid lines) compared to the respective (same area) background distributions (shaded regions).

may have already been dispersed into the background along dynamical evolution.

To be comprehensive in the interpretation of proper motion data in the context of determining the physical nature of an object, one must include the proper motion of faint stars as above together with those for the brighter stars. In this sense, 5 of the 6 bright stars (a clump of giants around $(J-H) \approx 0.48$ and $J \approx 8.2$ ) in the CMD of NGC 2180 have similar proper motions, within uncertainties, as can be seen in Table 2. The deviant proper motion of the first star might be accounted for by binary motion affecting its proper motion (compare to Fig. 9, bottom panel).

We conclude that, although there is a reduced number of stars in the field of NGC 2180, the proper motion properties are consistent with the presence of binaries superimposed on a normal distribution for single stars.

\section{Concluding remarks}

As a consequence of the internal dynamical evolution and the relentless Galactic tidal pull, most open clusters are expected to evaporate completely in less than $1 \mathrm{Gyr}$, leaving behind a remnant with characteristics that depend on the cluster's initial conditions. Only the more massive open clusters may survive to old ages. In this context, the observation of an actually dissolving open cluster is very interesting to check existing theories on dynamical evolution of stellar systems, $N$-body codes in particular, as well as to test stellar evolution theories. 
Table 2. Proper motions for the bright stars in NGC 2180.

\begin{tabular}{cccrr}
\hline \hline $\begin{array}{c}\alpha(2000) \\
(\mathrm{hms})\end{array}$ & $\begin{array}{c}\delta(2000) \\
\left({ }^{\prime \prime \prime \prime}\right)\end{array}$ & $\begin{array}{c}P M(\alpha) \times \cos (\delta) \\
(\mathrm{mas} / \mathrm{yr})\end{array}$ & $\begin{array}{r}P M(\delta) \\
(\mathrm{mas} / \mathrm{yr})\end{array}$ & $\begin{array}{r}P M(\operatorname{modulus}) \\
(\mathrm{mas} / \mathrm{yr})\end{array}$ \\
\hline $06: 09: 19.63$ & $+04: 45: 23.3$ & $-30.7 \pm 1.2$ & $-5.4 \pm 1.2$ & $31.2 \pm 11.2$ \\
$06: 09: 37.97$ & $+04: 44: 15.4$ & $+1.4 \pm 1.5$ & $-3.7 \pm 1.4$ & $4.0 \pm 11.4$ \\
$06: 09: 39.43$ & $+04: 47: 18.2$ & $-1.2 \pm 1.1$ & $-0.5 \pm 1.1$ & $1.3 \pm 11.1$ \\
$06: 09: 51.40$ & $+04: 51: 13.5$ & $+2.1 \pm 2.0$ & $-9.5 \pm 2.1$ & $9.7 \pm 12.0$ \\
$06: 09: 53.58$ & $+04: 55: 34.5$ & $-1.5 \pm 1.4$ & $-3.5 \pm 1.4$ & $3.8 \pm 11.4$ \\
$06: 10: 08.03$ & $+04: 51: 29.0$ & $+3.5 \pm 1.4$ & $-1.9 \pm 1.5$ & $4.0 \pm 11.5$ \\
\hline
\end{tabular}

Notes. The proper motion modulus in Col. 5 is given by $P M$ (modulus) $=\sqrt{P M(\alpha)^{2} \times \cos (\delta)^{2}+P M(\delta)^{2}}$.

In the present work we analysed the physical structure, stellar content and dynamical state of the intermediate-age open cluster NGC 3680, formerly considered to be in the last stages of its dynamical evolution. We also examined in detail the overlooked object NGC 2180. The present analyses make use mostly of $J$ and $H$ 2MASS All Sky data release photometry.

NGC 2180 presents a non-uniform radial distribution of stars (Sect. 4), with $1-\sigma$ Poisson error bars due to the small number of member stars. Its radial density profile appears to have a central concentration of stars, as well as a corona (Fig. 4). From a King law fit we estimate $R_{\text {core }}=0.7 \pm 0.3 \mathrm{pc}$ and a linear limiting diameter of $9.5 \pm 1.2 \mathrm{pc}$. Its $M_{J} \times(J-H)$ CMD (Fig. 5) is depleted of stars near the turnoff, and can be fitted with $710 \mathrm{Myr}$ isochrones of solar and sub-solar metallicity. The $\left[Z / Z_{\odot}\right]=0.0$ solution results in $(m-M)_{0}=10.10 \pm$ $0.20, E(B-V)=0.0$ and a distance to the Sun $d_{\odot}=1.05 \pm$ $0.08 \mathrm{kpc}$, while the $\left[Z / Z_{\odot}\right]=-0.4$ solution gives $(m-M)_{0}=$ $9.40 \pm 0.20, E(B-V)=0.18$ and $d_{\odot}=0.76 \pm 0.06 \mathrm{kpc}$. Thus, we adopt as a distance to the Sun $d_{\odot}=0.91 \pm 0.15 \mathrm{kpc}$, which puts NGC 2180 at a galactocentric distance of $8.8 \pm 0.1 \mathrm{kpc}$. Mass segregation and advanced Galactic tidal stripping on NGC 2180 may be inferred by the spatial properties of its LFs (Fig. 6). Low-mass stars seem to be severely depleted from the MS in each region, from the center to the cluster's limiting radius. In addition, the MS of the corona, although depleted as well, is slightly more populated by low-mass stars than the MS of more internal regions. We infer the observed stellar mass in NGC 2180 as $\sim 47 \pm 7 M_{\odot}$.

NGC 3680 presents a uniform radial distribution of stars, with a well-defined core and a corona, with $R_{\text {core }}=0.7 \pm$ $0.1 \mathrm{pc}$ and a linear limiting diameter of $12.8 \pm 1.3 \mathrm{pc}$ (Sect. 4 ). Its $M_{J} \times(J-H) \mathrm{CMD}$ (Fig. 5) presents a nearly complete MS, including the turnoff and giants. We derive an age of $\tau_{\text {age }} \approx 1.6 \mathrm{Gyr},(m-M)_{0}=10.00 \pm 0.20, E(B-V)=0.00$ and $d_{\odot}=1.00 \pm 0.09 \mathrm{kpc}$, in reasonable agreement with previous works. The LF of the central region of NGC 3680 (Fig. 6) is depleted of low-mass stars which, contrary to what is observed in NGC 2180, are still present in the external region and corona. Thus, Galactic tidal stripping has not yet been effective in severely depleting NGC 3680 of stars. For NGC 3680, a MF fit $\phi(m) \propto m^{-(1+\chi)}$ resulted in a slope $\chi=2.06 \pm 1.08$, and in an observed stellar mass (MS and giants) of $\approx 130 \pm$ $24 M_{\odot}$. Extrapolating the MF fit down to the theoretical lowmass end $m_{\text {low }}=0.08 M_{\odot}$, the total stellar mass in NGC 3680 turns out to be $\sim(2.4 \pm 1.2) \times 10^{3} M_{\odot}$, which agrees with previous estimates, within uncertainties. Assuming a more representative IMF (Kroupa 2001), which flattens for masses below $\sim 0.5 M_{\odot}$, the total stellar mass in NGC 3680 turns out to be $546 \pm 206 M_{\odot}$.

Finally, comparing NGC 2180 and NGC 3680 with clusters in other dynamical states, as well as open cluster remnants, we found that the less-massive nature of NGC 2180 puts it closer to cluster remnants than NGC 3680 .

The above arguments suggest that NGC 2180 is in a more advanced dynamical state than NGC 3680, on its way to becoming a fossil cluster. Thus, NGC 2180 may be the missing link between evolved open clusters and remnants.

Acknowledgements. We thank the referee, Dr. R. de Grijs, for interesting remarks. This publication makes use of data products from the Two Micron All Sky Survey, which is a joint project of the University of Massachusetts and the Infrared Processing and Analysis Center/California Institute of Technology, funded by the National Aeronautics and Space Administration and the National Science Foundation. We employed catalogues from CDS/Simbad (Strasbourg) and Digitized Sky Survey images from the Space Telescope Science Institute (US Government grant NAG W-2166) obtained using the extraction tool from CADC (Canada). We also made use of the WEBDA open cluster database. We acknowledge support from the Brazilian Institution CNPq.

\section{References}

Alter, G., Ruprecht, J., \& Vanysek, V. 1970, in Catalogue of star clusters and associations + supplements, 2nd edition (Budapest: Akad. Kiado)

Anthony-Twarog, B. J., Twarog, B. A., \& Shodhan, S. 1989, AJ, 98, 1634

Anthony-Twarog, B. J., Twarog, B. A., Heim, E. A., \& Caldwell, N. 1991, AJ, 102, 1056

Bergond, G., Leon, S., \& Guibert, J. 2001, A\&A, 377, 462

Bica, E., Santiago, B. X., Dutra, C. M., et al. 2001, A\&A, 366, 827

Bica, E., Bonatto, C. J., \& Dutra, C. M. 2003, A\&A, 405, 991

Binney, J., \& Tremaine, S. 1987, in Galactic Dynamics (Princeton, NJ: Princeton University Press)

Binney, J., \& Merrifield, M. 1998, in Galactic Astronomy (Princeton, NJ: Princeton University Press), Princeton series in astrophysics

Bonatto, C., Bica, E., \& Girardi, L. 2004, A\&A, 415, 571

Bonatto, C., \& Bica, E. 2003, A\&A, 405, 525

Bonatto, C., Bica, E., \& Santos, J. F. C., Jr. 2004, A\&A, in preparation 
Bruntt, H., Frandsen, S., Kjeldsen, H., \& Andersen, M. I. 1999, Montgomery, K. A., Marschall, L. A., \& Janes, K. A. 1993, AJ, 106, A\&AS, 140, 135

Carraro, G. 2002, A\&A, 385, 471 181

Dias, W. S., Alessi, B. S., Moitinho, A., \& Lépine, J. R. D. 2002, A\&A, 389, 871

Dreyer, J. L. E. 1888, MmRAS, 49, 1

Dutra, C. M., Santiago, B. X., \& Bica, E. 2002, A\&A, 381, 219

Eggen, O. J. 1969, ApJ, 155, 439

Friel, E. D. 1995, ARA\&A, 33, 381

de la Fuente Marcos, R. 1996, A\&A, 314, 456

de la Fuente Marcos, R. 1997, A\&A, 322, 764

de la Fuente Marcos, R. 1998, A\&A, 333, L27

Girardi, L., Bertelli, G., Bressan, A., et al. 2002, A\&A, 391, 195

von Hippel, T., \& Sarajedini, A. 1998, AJ, 116, 1789

Houston, W. S. 1976, S\&T, 51, 68

King, I. 1966, AJ, 71, 64

Nissen, P. E. 1988, A\&A, 199, 146

Nordström, B., Andersen, J., \& Andersen, M. I. 1996, A\&AS, 118, 407

Pavani, D. B., Bica, E., Dutra, C. M., et al. 2002, A\&A, 374, 554

Pavani, D. B., Bica, E., Ahumada, A. V., \& Clará, J. J. 2003, A\&A, 399, 113

Perryman, M. A. C., Brown, A. G. A., Lebreton, Y., et al. 1998, A\&A, 331,81

Scalo, J. 1998, in The Stellar Initial Mass Function, ed. G. Gilmore, \& D. Howell (San Francisco: ASP), ASP Conf. Ser., 142, 201

Skrutskie, M., Schneider, S. E., Stiening, R., et al. 1997, in The Impact of Large Scale Near-IR Sky Surveys, ed. Garzon et al. (Netherlands: Kluwer), 210, 187

Kozhurina-Platais, V., Girard, T. M., Platais, I., \& van Altena, W. F. 1997, AJ, 109, 672

Kroupa, P. 2001, MNRAS, 322, 231

Kroupa, P., Tout, C. A., \& Gilmore, G. 1991, MNRAS, 251, 293

Lyngå, G. 1987, in Computer based catalogue of open cluster data, 5th ed. (Strasbourg: CDS)

Mazzei, P., \& Pigatto, L. 1988, A\&A, 193, 148

Mermilliod, J. C. 1996, in The origins, evolution, and destinies of binary stars in clusters, ASP Conf. Ser., 90, 475

Soares, J. B., \& Bica, E. 2002, A\&A, 388, 172

Sulentic, J. W., \& Tifft, W. G. 1973, in The Revised New General Catalogue of Nonstellar Astronomical Objects (RNGC) (Tucson, Arizona:University of Arizona Press)

Takahasi, K., \& Portegies Zwart, S. F. 2000, ApJ, 535, 759

Twarog, B. A., Ashman, K. M., \& Anthony-Twarog, B. J. 1997, AJ, 114,2556

Wielen, R. 1991, in The Formation and Evolution of Star Clusters, ed. K. Janes, ASP Conf. Ser., 13, 343

Zacharias, N., Urban, S. E., Zacharias, M. I., et al. 2004, AJ, 127, 3043 